\title{
Dynamic bonding of metallic nanocontacts: Insights from experiments and atomistic simulations
}

\author{
M. A. Fernández, ${ }^{1}$ C. Sabater, ${ }^{1,2}$ W. Dednam,,${ }^{1,3}$ J. J. Palacios, ${ }^{4}$ M. R. Calvo, ${ }^{1,5}$ C. Untiedt, ${ }^{1}$ and M. J. Caturla ${ }^{1, *}$ \\ ${ }^{1}$ Departamento de Física Aplicada, Universidad de Alicante, San Vicente del Raspeig, E-03690 Alicante, Spain \\ ${ }^{2}$ Huygens-Kamerlingh Onnes Laboratorium, Leiden University, Niels Bohrweg 2, 2333 CA Leiden, Netherlands \\ ${ }^{3}$ Department of Physics, Science Campus, University of South Africa, Private Bag X6, Florida Park 1710, South Africa \\ ${ }^{4}$ Departamento de Física de la Materia Condensada, Instituto de Ciencia de Materiales Nicolás Cabrera (INC), and Condensed Matter \\ Physics Center (IFIMAC), Universidad Autonoma de Madrid, Cantoblanco, 28049 Madrid, Spain \\ ${ }^{5}$ CIC NanoGune, E-20018, Donostia, San Sebastian, Spain
}

(Received 13 November 2015; revised manuscript received 29 January 2016; published 26 February 2016)

\begin{abstract}
The conductance across an atomically narrow metallic contact can be measured by using scanning tunneling microscopy. In certain situations, a jump in the conductance is observed right at the point of contact between the tip and the surface, which is known as "jump to contact" (JC). Such behavior provides a way to explore, at a fundamental level, how bonding between metallic atoms occurs dynamically. This phenomenon depends not only on the type of metal but also on the geometry of the two electrodes. For example, while some authors always find $\mathrm{JC}$ when approaching two atomically sharp tips of $\mathrm{Cu}$, others find that a smooth transition occurs when approaching a $\mathrm{Cu}$ tip to an adatom on a flat surface of $\mathrm{Cu}$. In an attempt to show that all these results are consistent, we make use of atomistic simulations; in particular, classical molecular dynamics together with density functional theory transport calculations to explore a number of possible scenarios. Simulations are performed for two different materials: $\mathrm{Cu}$ and $\mathrm{Au}$ in a [100] crystal orientation and at a temperature of $4.2 \mathrm{~K}$. These simulations allow us to study the contribution of short- and long-range interactions to the process of bonding between metallic atoms, as well as to compare directly with experimental measurements of conductance, giving a plausible explanation for the different experimental observations. Moreover, we show a correlation between the cohesive energy of the metal, its Young's modulus, and the frequency of occurrence of a jump to contact.
\end{abstract}

DOI: 10.1103/PhysRevB.93.085437

\section{INTRODUCTION}

Metallic nanocontacts are of interest from fundamental and technological points of view. The conventional miniaturization of electronic devices is fast approaching its limits and new possibilities must be explored. A useful tool that can produce contacts down to a single atom is the scanning tunneling microscope (STM) [1]. There are manifold reasons to undertake a study of monatomic devices by means of a STM: it permits one to measure electronic transport through them, probe their mechanical and magnetic properties, and, very importantly, control and manipulate the few atoms in the devices themselves [2]. Besides its possible technological applications, metallic nanocontacts can be used to study how dynamic bonding between two metals occurs. In this paper we focus on the fundamental understanding of contact formation in metals.

Several groups have studied the process of contact formation by using either STMs or mechanically controllable break junctions (MCBJs) [3-6]. Measuring the conductance as two electrodes approach each other reveals, in certain situations, a jump in the conductance going from tunneling to contact regimes. This "jump to contact" (JC) phenomenon depends on the metal [3] but also on the geometry of the two electrodes $[4,5]$.

These experimental techniques, however, only provide indirect information, through conductance values, about how contact between the two electrodes occurs, but they do not permit real-time observation of the dynamics at the single-

\footnotetext{
*mj.caturla@ua.es
}

atom level. Therefore, theoretical modeling represents an important tool for shedding light on the processes that govern such systems. In this regard, classical molecular dynamics (CMD) simulations [7] provide the means to model the dynamic contact formation and rupture processes that take place during an STM experiment by allowing one to follow the time evolution of every atom in the system. In fact, it was through CMD studies that the abrupt changes in conductance observed experimentally in metallic systems was first understood [8] as changes in the number of atoms at the minimum cross section. However, in order to make a direct comparison with experiments possible, one must be able to calculate the conductance across the contacts as they are formed or ruptured. Hence, through a combination of molecular dynamics simulations and density functional theory (DFT) [9] transport calculations, STM experiments can be realistically modeled [3,10-12]. In this paper we show how, through the use of these simulation methods, it is possible to make qualitative as well as quantitative comparisons with atomic contact experiments performed in copper and gold.

Moreover, these simulations provide a description of the contribution of short- and long-range forces to contact formation in metals, as well as a way to infer the shape of an atomic contact from the behavior of the conductance at the point of contact [12].

The remainder of the paper is organized as follows: In Sec. II we describe the experimental techniques used to study the jump-to-contact phenomenon as well as related experimental results, including some new results. Then, in Sec. III, we discuss the theoretical techniques we have used to model the experiments and proceed to present our results and compare them with the experiments in Sec. IV. A discussion 
is presented in Sec. $\mathrm{V}$ where the mechanism for bonding under different conditions is explained. We finally conclude in Sec. VI.

\section{EXPERIMENTAL OBSERVATIONS OF JUMP TO CONTACT}

The most commonly used techniques for studying electron transport in atomic-sized contacts are scanning tunneling microscopy (STM) and mechanically controllable break junctions (MCBJs) [8]. Both methods provide information on electronic behavior in few- or single-atom contacts. In the experiments, a bias voltage (100 $\mathrm{mV}$ in our case) is applied across the leads and then the current or, more precisely, the conductance across the atomic constriction, is measured (usually in units of the quantum of conductance $G_{0}=2 e^{2} / h$ ). The aspect ratio of the atomic constriction (its length divided by its minimum cross section) [13], or the number of atoms in the constriction, can be changed by indenting to a greater or lesser extent; the indentation process is controlled by means of a piezo system in practice. The results of the electronic transport measurements are recorded as conductance $(G)$ versus piezo displacement (measured in volts applied to the piezo system, or in $\mathrm{nm}$ ) and are referred to as a conductance trace.

As an example, Fig. 1(a) shows two traces obtained via MCBJs at a low temperature $(4.2 \mathrm{~K})$ and in a cryogenic vacuum, for samples of $\mathrm{Cu}$ and $\mathrm{Au}$, as the two electrodes approach each other until a contact is formed. It is clear from these two curves that, right before contact, there is a discontinuity from the tunneling regime (around $0 G_{0}$ ) to $1 G_{0}$; that is, a jump to contact. These traces can be obtained in the experiment thousands of times and in the cases of $\mathrm{Au}$ and $\mathrm{Cu}$ a jump to contact always appears. However, this is not the case for all metals. In some metals, such as W, most traces show a smooth transition between tunneling current and contact [3].

The frequency of occurrence of jump to contact has been studied in many different metals with some such as $\mathrm{Au}, \mathrm{Cu}$, or Pt always exhibiting a jump to contact. Others such as $\mathrm{Ni}$, Co, or Ir exhibit jump to contact only between $70 \%$ and $80 \%$ of the time while for $\mathrm{W}$ most of the time jump to contact does not appear $[3,4,14,15]$. We gathered information about this frequency of occurrence of a jump to contact for twelve different metals and present here a correlation between this frequency and the values of cohesive energy and Young's modulus of the different materials. Figure 1(b) shows the value of the Young's modulus vs the cohesive energy for these metals. Colors represent the frequency of occurrence of jump to contact. In order to create Fig. 1(b), we plot three columns in a contour graph, with the $y$ axis containing the value of the Young's modulus [16] and the $x$ axis showing the value of the cohesive energy, as obtained from Kaptay et al. [17]. The color scale represents the values of the probability of jump to contact obtained from Sabater [14] and Calvo [15]. This plot shows that, at low cohesive energies, such as in the case of $\mathrm{Pb}$ or $\mathrm{Au}$, jump to contact always occurs, while at very high cohesive energies (W, for example) the transition from the tunneling regime to contact is smooth. But not only the cohesive energy plays a role in the appearance of a jump to contact; a correlation with Young's modulus also seems apparent in this graph. For the same cohesive energy, as Young's modulus increases, the probability of exhibiting jump to contact decreases (see, for example, $\mathrm{Pd}$ vs $\mathrm{Ni}$ or $\mathrm{Co}$, or the case of Ir).

All the experimental measurements presented in Fig. 1 were recorded as two tips approached each other. However, the phenomenon of jump to contact has also been observed for different geometries. Kröger et al. [4]. showed that in $\mathrm{Cu}$ a jump to contact exists when an STM tip approaches an atomically flat surface. Interestingly, just the presence of a single adatom on the surface makes the transition from tunneling to contact smooth. This seems to indicate that not only the type of metal but also the geometry of the tip determines how contact is going to occur.

\section{SIMULATION METHODS}

In order to understand the experimental results described above and in an attempt to explain why similar techniques give rise to different results, while at the same time trying to uncover
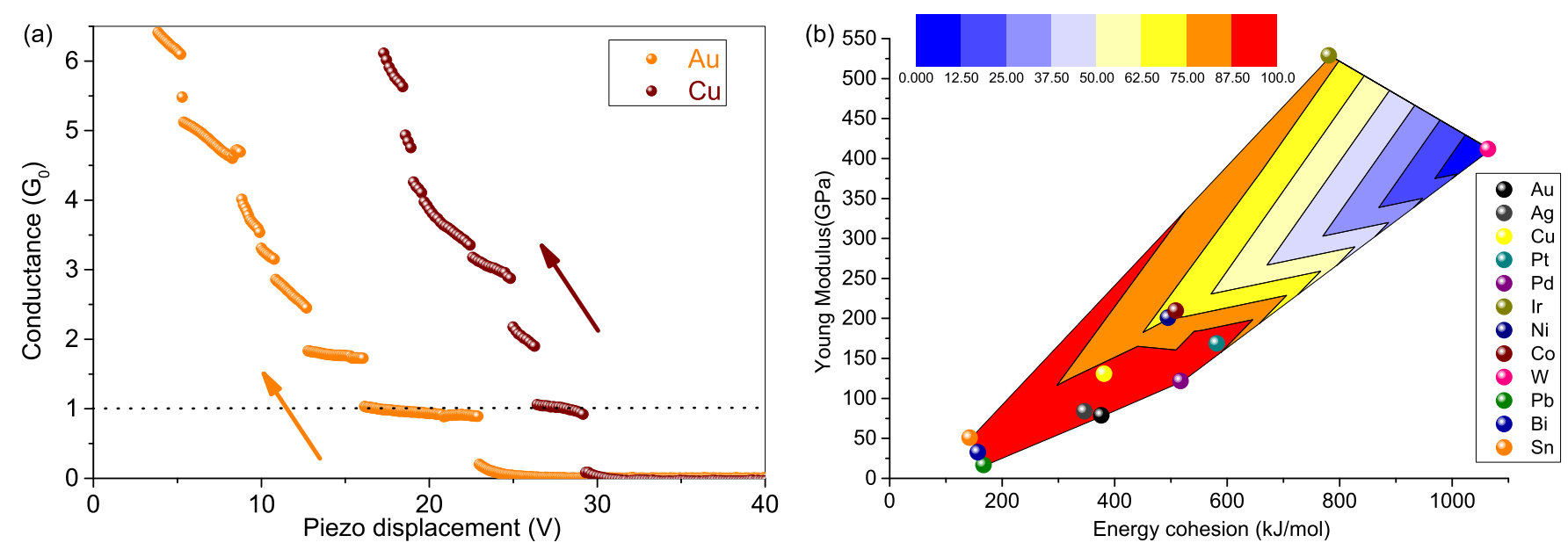

FIG. 1. (a) Conductance traces obtained by using the MCBJ technique during the formation of a $\mathrm{Au}$ and a $\mathrm{Cu}$ nanocontact at $4.2 \mathrm{~K}$. The arrows indicate the direction in the measurement of conductance, from out of contact ( 0 conductance), to first point of contact ( $\left.\sim 1 G_{0}\right)$, and beyond. (b) Correlation between cohesive energy, Young's modulus and frequency of appearance of jump to contact for several metals. 
the mechanisms at play, we resort to a combination of two different methods: atomistic molecular dynamics to simulate the geometric evolution of the atomic-sized contacts, and $a b$ initio calculations based on DFT to obtain the conductance and thus permit a direct comparison with the experiments.

Classical molecular dynamics simulations solve Newton's second law for all the particles in the system that is the object of study by using empirical potentials [7]. One of the key advantages of CMD is that it allows the classical trajectories of all the particles in the system to be visualized at any moment and followed for the entire time evolution of the system. Another advantage of CMD is that the system can be made up of as few as hundreds up to as many as millions of particles, which would be prohibitively expensive in terms of computational effort in the case of more rigorous first principles quantum methods.

We confine our study to nanosized electrodes made of the noble metals $\mathrm{Au}$ and $\mathrm{Cu}$ and consider different shapes and the [100] crystallographic orientation. These metals are monovalent and therefore likely to exhibit conductance quantization, which facilitates the study of the JC phenomenon because, in theory, there is only one eigenchannel available per atom for electronic transmission through the minimum cross section of the interacting electrodes. Thus, we would expect to see the conductance increase gradually from the tunneling regime to a value of roughly $1 G_{0}$ if the contact were monatomic and there were no JC. Conversely, one would expect to see a jump from tunneling currents to roughly one unit of conductance if there were a JC and there were only a single atom in the minimum cross section.

From the point of view of molecular dynamics, bulk noble metals are also rather simple to model because the potential used to describe the interactions between the atoms is scalar and isotropic in space, i.e., the bonding is nondirectional. A useful semi-empirical potential that effectively describes the interatomic interactions in CMD simulations is the embedded atom method (EAM) potential [18]. It consists of a sum of two contributions to the interaction energy: a pair-wise energy term and the so-called embedding term, which represents the energy that is needed to embed an atom in an electron gas at a given lattice site, for a given value of the electronic density at that lattice site. An EAM-type potential that works particularly well in the case of noble metals is the one described in Zhou et al. [19]. It has been successfully fit to a number of basic material parameters of the metals, including lattice constants, elastic constants, bulk moduli, vacancy formation energies, sublimation energies, and heats of solution. Nevertheless, one should keep in mind the limitations of these potentials when applied to low coordination systems.

We employ the widely used open-source code Large-Scale Atomic/Molecular Massively Parallel Simulator (LAMMPS) to carry out our simulations [20,21]. We simulate at the boiling temperature of liquid helium, $4.2 \mathrm{~K}$, which is commonly reached in low-temperature contact experiments [8]. The temperature is kept constant in the simulation through the use of a Nose-Hoover thermostat [22,23].

The strain rate of the simulations, or speed of approach between the two electrodes, is significantly larger than those used experimentally, which is one of the limitations of CMD simulations. However, the speeds simulated are always much lower than the speed of sound in these materials and are typical for molecular dynamics simulations [8,24]. At the low temperatures considered, diffusion effects are negligible and one can expect that equilibrium is reached in the short time of the simulations. To complete our simulations in a reasonable amount of time, we use systems containing at most a few thousand atoms. Likewise, the speed of approach and retraction between the surfaces, about $0.4 \mathrm{~m} / \mathrm{s}$, is fast enough to make the simulations run for only as long as needed, but realistic enough to ensure that equilibrium thermodynamics still apply [24].

The surfaces are moved by freezing the top and bottom layers and displacing them at the above constant rate; the remaining atoms responding dynamically to the rigid motion of the frozen layers. In the experiments, the smallest displacement that can be achieved between conductance readings amounts to a few picometers, or at the very smallest, a fraction of a picometer [25]. This corresponds to recording time frames every few thousand femtoseconds during the simulations. In our case, we write system coordinates every $100 \mathrm{fs}$, which corresponds to a displacement of $0.04 \mathrm{pm}$.

Simulations consist of tips oriented along the [100] crystallographic direction. Two types of simulations are performed. On the one hand a sharp tip is moved toward an atomically flat surface oriented along the [100] direction. In this particular type of simulation we study the tip approaching a hollow site on the surface, an atom in the surface, or an adatom placed in the most stable position on the surface. These simulations mimic the experiments performed by Kröger et al. [4,5]. The second type of simulation consist of approaching two tips formed by repeated contact between two electrodes, following the experimental setup of Untiedt et al. [3] and Trouwborst et al. [6]. In this type of simulation, a structure of an initially neck-like shape (that mimics the notched wire commonly encountered in MCBJ experiments) is stretched until it breaks, by pulling the top and bottom layers apart at $0.4 \mathrm{~m} / \mathrm{s}$. Once the nanocontact breaks it is again brought into contact in a process known as mechanical annealing. The whole process is repeated several times until a stable structure is obtained, which generally consists of two very sharp tips, which are then considered as our initial structures to study the phenomenon of jump to contact. Details about these simulations can be found in the supplementary material [26] and in Sabater et al. [11] and Dednam et al. [27].

In order to understand how contact occurs, we usually plot the traces of distance between electrodes obtained from all these CMD simulations. The way we do this is by initially determining the two atoms which are the first ones to make contact when approaching both surfaces and then record their distance (vertical component) during the development of the complete simulation.

As a clear demonstration of the ability of our simulations to reproduce experimental results, we calculate the minimum force needed to break a gold nanowire. The result we find, $1.5 \mathrm{nN}$, is in striking agreement with the experimental results reported by Rubio et al. [28]. Moreover, our simulations also reproduce an equilibrium distance between the two atoms in the gold dimer that is in very good agreement with the result of $2.5 \pm 0.2 \AA$ obtained by Trouwborst et al. [6]. Details about this calculation can be found in supplementary material [26]. 

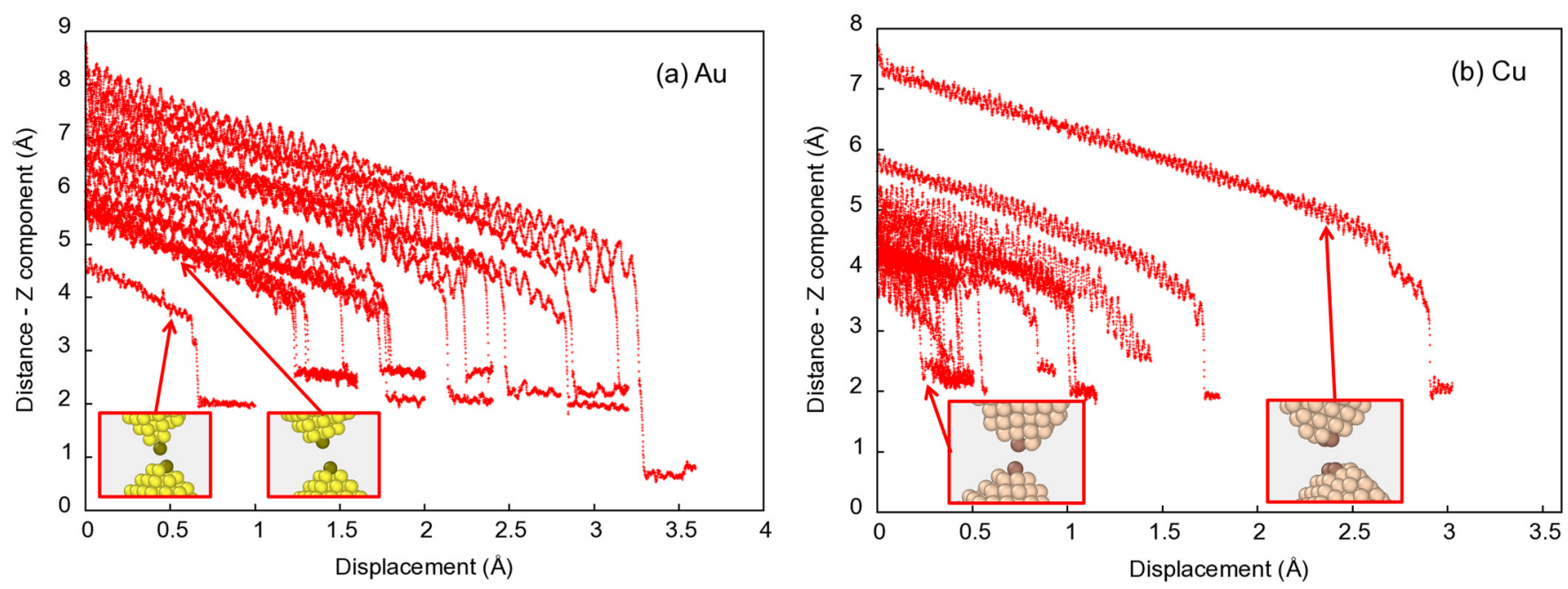

FIG. 2. Traces of tip-to-tip distance for mechanical annealing of (a) $\mathrm{Au}$ and (b) $\mathrm{Cu}$. The left inset in panel (a) shows the tip geometries just before first contact after one cycle of annealing, while the right inset shows the corresponding structure after 19 cycles. In panel (b), the right inset shows the structure just before first contact after a single cycle, and the left inset shows the corresponding tip geometries after 19 cycles.

Finally, to permit a direct comparison with the experiments, we need to be able to calculate conductance. By its very nature, CMD cannot provide such information, because there are no electrons present in the simulations (their effect is contained in the semi-empirical potential that describes the interactions between the metal atoms in the simulations). Therefore, a quantum-mechanical approach is required, the details of which we discuss in the next section.

In order to calculate the conductance of the structures taken from molecular-dynamics-simulation trajectories, we have used the electronic transport code ANT.G [29-32], which depends on DFT parameters calculated by GAUSSIAN09 [33]. The structures obtained via CMD contain around 3000 atoms. In order to compute the conductance of these structures via DFT calculations we have reduced the region of interest to around 500 atoms, keeping only those atoms that lie in a box smaller than the original simulation domain and centered on the region of first contact, or minimum cross section. However, in order to be accurate in the calculations of the conductance we have opted to assign a larger basis set of 11 valence electrons to 40 atoms in the contact region. The rest of the atoms were assigned a basis set of one valence electron.

The molecular dynamics simulations run for approximately $1.2 \mathrm{~ns}$, during which the positions and velocities of the atoms are updated every 1 fs by the standard Verlet integration algorithm in LAMMPS $[20,21]$. Thus, for the conductance calculations, we take time frames from the CMD simulation trajectories starting at $t=200 \mathrm{ps}$ and every $200 \mathrm{ps}$ thereafter up until the moment at which the tip and surface are about to make contact. At this point, we take CMD snapshots at much shorter intervals to capture the behavior of the conductance as the electrodes come into contact with each other. We continue the calculations for a few additional snapshots after first contact, where the conductance values persist at or above $1 G_{0}$. This permits the construction of conductance traces such as those obtained experimentally; for example, in Untiedt et al. [3] and Kröger et al. [4,5].

\section{SIMULATION RESULTS}

Figure 2 shows the distance traces obtained for the simulations of mechanical annealing [11], or work hardening, of $\mathrm{Au}$ and $\mathrm{Cu}$ electrodes. Each trace is the result of a simulation of two electrodes approaching each other until contact. The insets show the configurations of the electrodes before contact, in one case after only one cycle of annealing, and in the other after 19 annealing cycles. Clearly, in all the cases there is a sharp drop in the distance between the two electrodes right before contact. This sharp jump is what we consider is related to the presence of a jump in the conductance, as we explain below. The sharp changes in distance are then in agreement with the experimental observation of jump to contact in $\mathrm{Au}$ and $\mathrm{Cu}$ when two sharp tips are considered $[3,15]$.

Note that, after 19 cycles of mechanical annealing, both the $\mathrm{Cu}$ (right panel, left inset) and $\mathrm{Au}$ (left panel, right inset) tips are very sharp, ending in a single atom. Only in the case of $\mathrm{Cu}$ are the tips initially (right panel, right inset) more rounded than sharp. Moreover, in both cases the two tips are slightly off center with respect to the vertical because, before breaking, the $\mathrm{Au}$ and $\mathrm{Cu}$ nanowires initially stretch and narrow down through the formation of [111]-oriented slip planes [34]. Therefore, cyclic loading occurs through slip planes sliding past each other so that the atoms at the apices of the tips do not end up making contact head-on, but instead sidelong.

The oscillations present in Fig. 2 are due to the thermostatting, which is applied every $100 \mathrm{fs}$, but also some lower-frequency oscillations exist due to a slight displacement of the atoms at the tip. These oscillations are, therefore, larger for the case of two tips than for the case of a tip and a surface. Moreover, these oscillations are reduced in $\mathrm{Cu}$ with respect to Au due to the rigidity of the former, as explained below.

The second type of simulations performed consist of an atomically sharp $\mathrm{Au}$ or $\mathrm{Cu}$ tip approaching a flat $\mathrm{Au}$ or $\mathrm{Cu}$ surface, respectively (see Fig. 3). Results are presented for three different situations: a tip approaching a hollow site on the surface (blue curve), a tip approaching directly an atom 

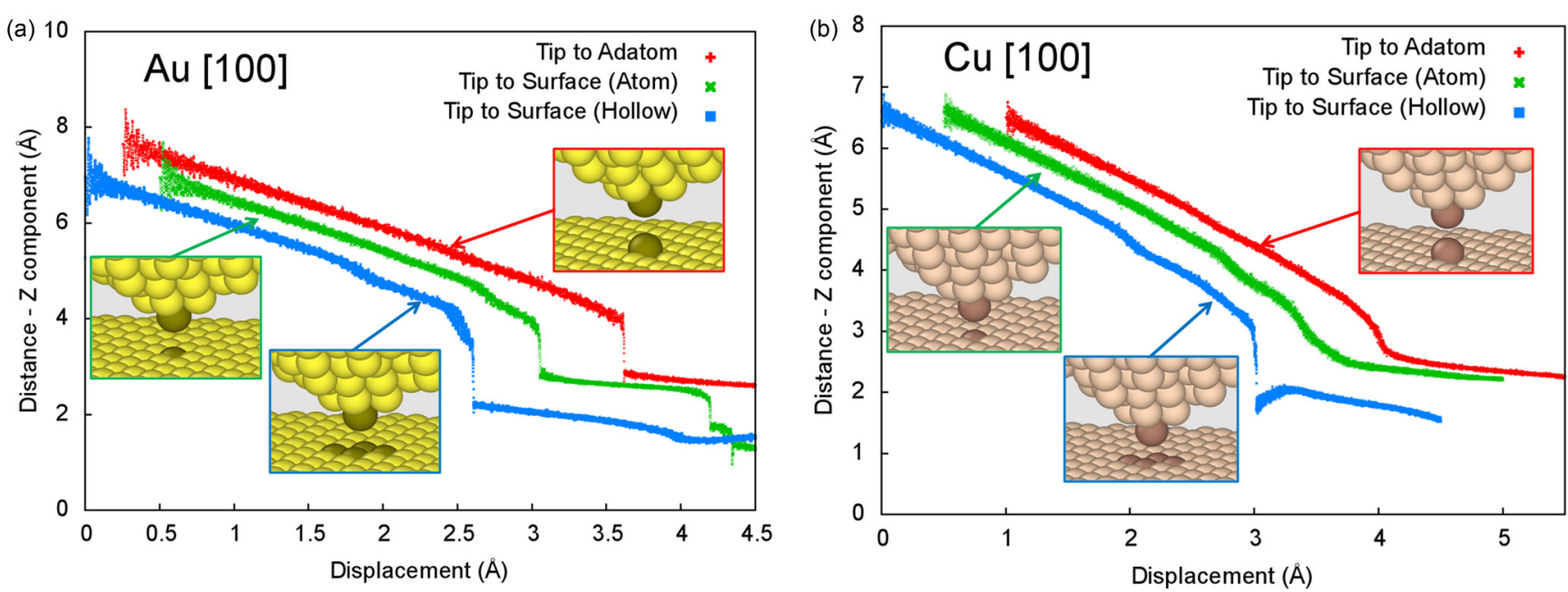

FIG. 3. CMD simulations for (a) Au and (b) Cu, oriented along the [100] direction. Distance between electrodes ( $Z$ component) versus displacement (both in $\AA$ ) for a sharp tip approaching a surface. Three different scenarios: tip aligned with surface atom (green curve), tip aligned with fourfold hollow (blue), and tip aligned with adatom lying in hollow (red). The traces have been shifted horizontally by $0.25 \AA$ in $\mathrm{Au}$ and by $0.5 \AA$ in $\mathrm{Cu}$. Inset images obtained with scientific software package Open Visualization Tool OVITO [35,36].

in the surface (green curve) and a tip approaching an adatom on the surface (red curve). The adatom has been located in the minimum energy configuration of all possible sites on the surface (a fourfold hollow). The insets in Fig. 3 show an image of the atomic configuration in the three different cases before contact. Note that the curves have been shifted sideways along the horizontal axis, by $0.25 \AA$ in the case of $A u$ and by $0.5 \AA$ in the case of $\mathrm{Cu}$, to be able to show more clearly the transition to contact in all three cases.

Here some striking differences can be observed between $\mathrm{Au}$ and $\mathrm{Cu}$ that were not present when approaching two sharp tips. In $\mathrm{Au}$, for all three cases, a sharp drop in distance is observed. However, in $\mathrm{Cu}$ a sharp jump is only observed in the case of the tip approaching a hollow site on the surface. When the tip approaches an adatom on or atom in the surface, the transition in the distance is very smooth. This seems to indicate that, in the case of $\mathrm{Cu}$, the geometry of the electrodes plays a very significant role. While two opposing sharp tips exhibit a sharp drop (see Fig. 2), a tip on an adatom shows a smooth transition. This behavior resembles the experimental observations of Kröger et al. [4] where, when approaching a STM tip to a surface, in some cases a smooth transition in the conductance is observed while in others there is a jump. Distance traces in Fig. 3 present some deviations from linear behavior before contact, which is related to the cutoff distance of the interatomic potential.

Movies from the CMD simulations for the case of tip to a surface atom (green curve) in $\mathrm{Au}$ and in $\mathrm{Cu}$ are available as supplementary material (Video 1 and Video 2, respectively) [26]. In the movies it is clearly shown that, in the case of Au (Video 1) [26], it is the atom at the tip that jumps towards the surface during the process of contact, although there is also a slight movement of the atom on the surface towards the tip. Note that not only the atom at the end of the tip moves, but also all its nearest neighbors. In Au, this relaxation of the tip as it approaches the surface is observed in all cases presented in Fig. 3(a), even when the tip is on top of an adatom (Video 3) [26]. In Cu, however, a smooth transition occurs for the case of tip-to-adatom and tip-to-surface-atom. As Video 2 shows [26], in $\mathrm{Cu}$ the tip is very rigid. Only in the case of a tip on top of a hollow site is a jump observed in $\mathrm{Cu}$ [blue curve, Fig. 3(b)], but the mechanism is quite different than in the case of Au. As Video 4 shows [26], the jump in this case is due to the detachment of the atom at the tip. As a result, due to the way the distance between tip and surface is calculated, the trace in this case upon contact shows first a decrease in the distance (when the atom detaches from the tip) and then an increase (when the detached atom is attracted again by the moving tip).

Figure 4 shows the conductance traces obtained in the case of $\mathrm{Au}$ and $\mathrm{Cu}$ and for the same cases shown in Fig. 3. The value of the conductance calculated from geometries obtained from the CMD simulations are plotted as a function of distance between the electrodes.

The results presented in Figs. 2 and 3 cannot be directly compared to the experimental observations because they are distance and not conductance traces. As mentioned above, we have performed DFT electronic transport calculations to obtain the conductance from the different configurations resulting from the CMD simulations.

By analogy with the sharp change in slope of the distance traces observed in Fig. 3 near the displacement when the two surfaces first come into contact, in the case of Au [see Fig. 4(a)] the conductance traces exhibit in all three cases a very sharp change in conductance at the same relative displacements between the electrodes as in Fig. 3(a). Here the traces are plotted in reverse with respect to the horizontal axis and the scale on the vertical axis has been chosen to be logarithmic to show the expected exponential dependence of current with the width of the tunnel barrier before the contact is established, such as in the experimental ones in Kröger et al. [4,5]. Note that the value of the conductance in all three scenarios of $\mathrm{Cu}$ and $\mathrm{Au}$ when the contact has been established is $\sim 1 G_{0}$, as expected for these metals and for contact through a single atom [37], and in agreement with the experimental data of Fig. 1 and others [38,39]. 

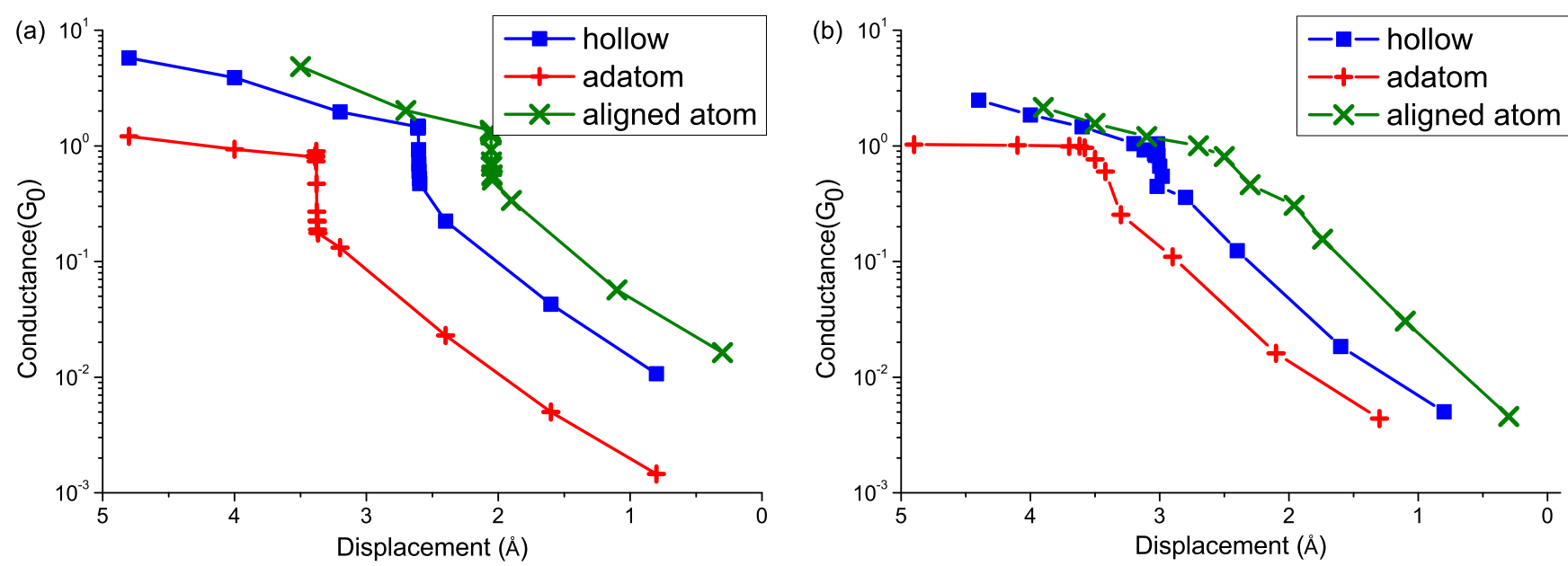

FIG. 4. Conductance traces obtained for the geometries of Fig. 3 for (a) Au and (b) Cu. Conductance (in units of $G_{0}$ ) versus displacement (A) for a sharp tip approaching a surface. Same three different scenarios as in Fig. 3. In panels (a) and (b), the trace "aligned-atom" has been shifted horizontally by $-0.5 \AA$. In panel (b), the trace "adatom" has been shifted horizontally by $+0.5 \AA$.

For copper the results are quite different between the different geometries and also reproduce the changes observed in the distance between tip and surface of Fig. 3(b). The change in distance is only sharp when the atom at the apex of the tip is aligned with a hollow site on the surface [see Fig. 3(b)]. This is also reflected by the sharp change in conductance in Fig. 4(b) for the hollow case, although it is smaller than the change in conductance obtained for Au. In Fig. 3(b), both the adatom and "aligned-atom" first-contact-distance traces are smooth, but the transition is smoother when the apex atom of the tip is aligned vertically with an atom in the surface below. In the case of $\mathrm{Cu}$, and as will be shown below, details in the geometry of the tip play a significant role in the presence or not of a jump to contact.

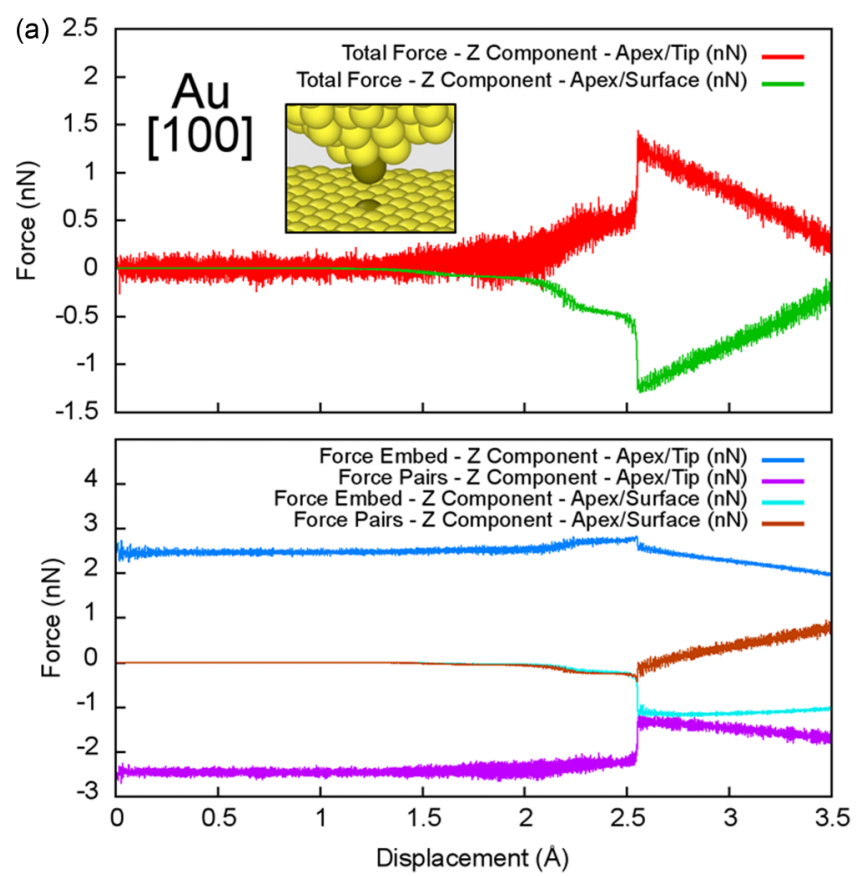

\section{DISCUSSION}

In order to understand the origin of the sharp changes in conductance or distance versus smooth transitions, we computed the force acting on the last atom of the tip of the electrode due to the other atoms within the tip, and the force on that same atom due to the atoms in the second electrode. Figure 5 shows the values of these forces for two cases, one case where there is a jump to contact [Fig. 5(a)] and one case where there is not a jump to contact [Fig. 5(b)]. In the first case, a sharp tip of Au approaches a flat surface with the tip located directly on top of an atom in the surface, showing the sharp drop in distance at the point of contact [green curve of Fig. 3(a)]. The jump in the force is clearly visible in Fig. 5(a).
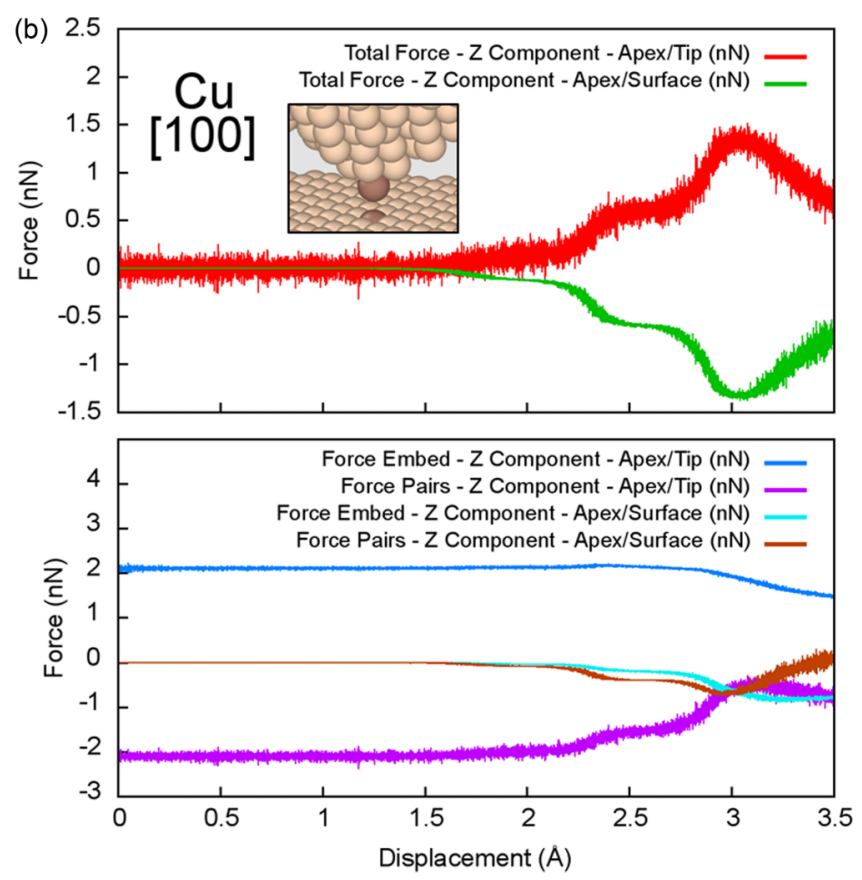

FIG. 5. Forces between the atom at the apex of the tip and the surface, and the atom at the apex and the rest of atoms in the tip, for the case of (a) $\mathrm{Au}$ and (b) $\mathrm{Cu}$. The same scenario has been chosen in both materials: sharp tip approaching a surface atom. 

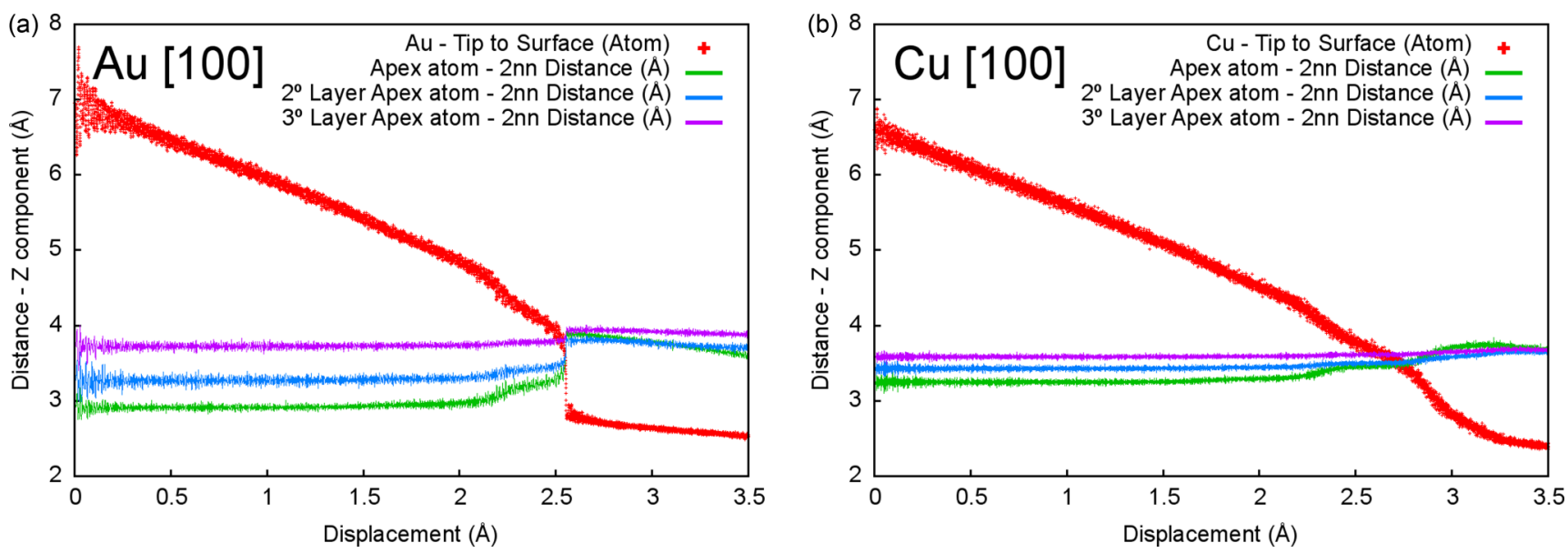

FIG. 6. Distance between atom at the tip, second layer and third layer to their second-nearest neighbors for (a) $\mathrm{Au}$ and (b) $\mathrm{Cu}$. Same scenario as in Fig. 5: sharp tip approaching a surface atom.

When the atom at the end of the tip [marked in dark in the inset of Fig. 5(a)] is located at a distance of about $3.7 \AA$ from the atom in the surface, it feels attracted towards the surface. A sharp change in the value of the force occurs at that point which is compensated by an upward force produced by the atoms in the tip of the electrode, such that the total force acting on the atom is still zero. This sharp change in the value of the force acting on the tip atom does not occur when there is not a jump to contact, as shown in Fig. 5(b). This figure corresponds to the case of a sharp $\mathrm{Cu}$ tip approaching a surface with the tip located on top of an atom of the surface [green curve of Fig. 3(b)]. Here the forces change gradually when the two electrodes approach each other and there is not a sharp change at any distance.

The forces on the atom at the tip have also been analyzed in terms of the component of the force due to the pair potential and due to the embedding energy of the EAM interatomic potential used in the calculations. In order to obtain the components of the force separately, several source files of LAMMPS have been modified: "bond_quartic," "compute_group_group," "compute_pair_local," "pair," "pair_eam," and "pair_hybrid." The bottom panel in Fig. 5(a) shows the force on the atom of the apex of the tip due to the rest of the atoms in the tip resulting from the pair potential (in purple) and the component of that force resulting from the embedding potential (in dark blue). When the tip is far away from the surface, the embedding force is an upward force, trying to keep the atom bound to the rest of the tip, while the pair potential gives rise to a downward force, or repulsive force between the atoms. As the tip approaches the surface there is only a very small change in the embedding force. However, there is a sharp change in the force due to the pair potential. The force decreases sharply at the distance of the jump to contact, resulting in the upward force shown in the top panel of Fig. 5(a). Shown in brown and light blue is the force between the tip and the surface from the pair potential and the embedding potential, respectively. When the tip and the surface are far apart, the force between tip and surface is zero, as expected. As the tip approaches the surface, we see in this case that the force due to the pair potential undergoes only a very small change while it is the force due to the embedding potential that experiences a sharp change, giving rise to the downward force shown in the top panel of Fig. 5(a). In the bottom panel of Fig. 5(b) we show also the different components in the case of $\mathrm{Cu}$ where there is not a jump to contact. Clearly there is a smooth transition in all the components of the force.

One important difference between the $\mathrm{Au}$ and $\mathrm{Cu}$ tips that is important to understand the dynamics of bonding is the relaxation occurring in a tip compared to the bulk structure. The second-nearest neighbor of the atom located at the apex of the tip of the electrode in $\mathrm{Au}$ is at a distance of $2.9 \AA$, significantly lower than the distance of second-nearest neighbors in bulk $\mathrm{Au}$ which is $4.08 \AA$. However, in the case of $\mathrm{Cu}$, the second-nearest neighbors are located at a distance of $3.2 \AA$ compared to the $3.6 \AA$ in the bulk. This means that Au experiences a contraction of about $29 \%$ in a sharp-tip configuration while $\mathrm{Cu}$ only has a contraction of about $12 \%$. This is due to the higher cohesive energy and therefore [40] embedding energy of Au with respect to $\mathrm{Cu}$ in this parametrization of the potential, which tries to maximize the electronic density by reducing the distance to the second-nearest neighbors. As a consequence, when the Au tip approaches the surface, it is able to relax more than in the case of $\mathrm{Cu}$, resulting in a sharper jump. This is also due to the lower Young's modulus of $\mathrm{Au}$ with respect of $\mathrm{Cu}$ (79 and $130 \mathrm{GPa}$, respectively). $\mathrm{Cu}$ tips are much stiffer than Au tips and therefore much less likely to undergo a sharp jump than Au. This can be seen in Fig. 6 where we plot the distance of the atom at the apex of the tip to its second-nearest neighbor as it approaches the surface. The distance between an atom in the second layer of the tip to its second-nearest neighbor, and between an atom in the third layer and its second-nearest neighbor is also plotted in the same graph. Figure 6(a) shows the results for $\mathrm{Au}$ in the case of a sharp tip approaching a surface directly on top of an atom of the surface [that is, the case of Fig. 5(a) shown above], and Fig. 6(b) is for $\mathrm{Cu}$ under the same conditions [like Fig. 5(b) above]. Clearly, Au is able to relax as it approaches the surface, changing its distance from $2.9 \AA$ when far away from the surface to the bulk value upon contact. For $\mathrm{Cu}$ however, the relaxation is very small, as explained above. The stiffness of the $\mathrm{Cu}$ tip does not allow for a sharp jump to contact while it does in Au. We should mention that simulations performed in $\mathrm{Cu}$ with less-symmetric tips than 
those presented here do show sharp jumps, since atoms at the tip can relax as they approach the surface.

The simulations presented here show that, for both $\mathrm{Cu}$ and $\mathrm{Au}$, when approaching two atomically sharp tips (Fig. 2), an abrupt change in the conductance is always observed at contact which is in good agreement with the experimental observations of MCBJ-like experiments [3,14,15]. Moreover, these simulations explain the correlation between Young's modulus and cohesive energy observed experimentally for the occurrence of jump to contact in many materials, shown in Fig. 1.

The behavior of the conductance traces when approaching a tip to a flat surface is, however, more complex. Our simulations show that, in $\mathrm{Au}$, an atomically sharp tip in contact with a surface or a surface with an adatom always presents a sharp change in the conductance [see Figs. 3(a) and 4(a)]. As explained above, this is due to the relaxation suffered by the tip. $\mathrm{Cu}$, on the other hand, shows a smooth transition in the conductance when approaching an adatom or when aligned with an atom of the surface, and only in the case of a tip on top of a hollow site on the surface is a sharp transition observed [see Figs. 3(b) and 4(b)].

Experimentally, a smooth transition is always observed for tip-adatom contact for the case of $\mathrm{Cu}$ (see review by Kröger et al. [4]), but not for the case of $\mathrm{Au}$ [5], in good agreement with our simulations, although experiments were performed using a surface oriented along the [111] direction. The explanation given for that behavior, in $\mathrm{Cu}$, is the enhanced elastic constant of the adatom. We calculated the elastic constant of an atom from the surface and an adatom in two different orientations, [100] and [111], following the procedure of Limot et al. [41]; that is, the atom is separated $4 \mathrm{pm}$ from the surface and the force on that atom is calculated. Table I shows the results obtained from our embedded-atom calculations compared to the DFT data of Limot et al. [41]. Indeed, the interatomic potential also reproduces an enhanced elastic constant for the adatom, with values in reasonable agreement with the DFT results.

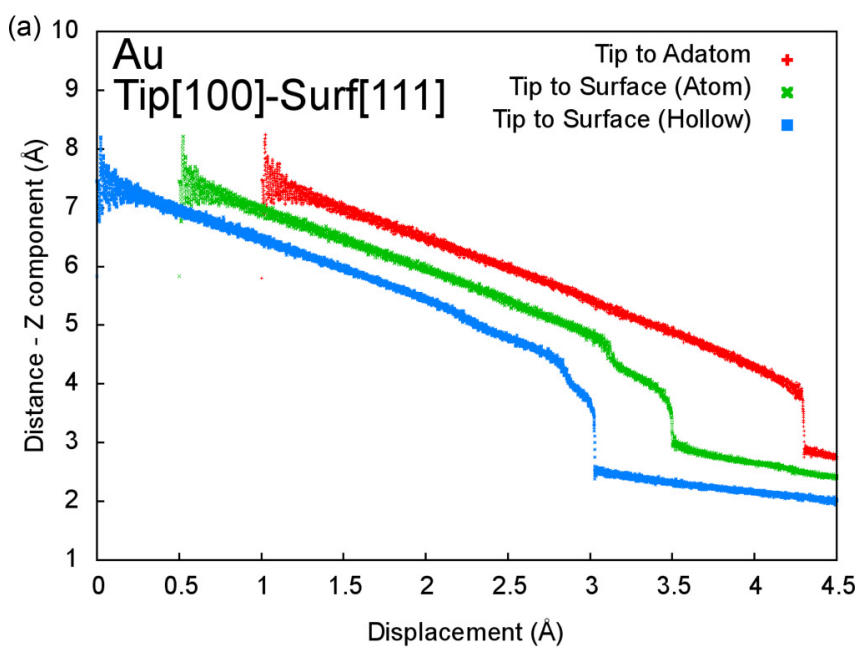

TABLE I. Values of the elastic constant of an atom at the surface and an adatom from $\mathrm{Cu}$ and $\mathrm{Au}$ and two different crystallographic orientations [100] and [111] obtained following the procedure of Limot et al. [41] and compared with available DFT data.

\begin{tabular}{lcclll}
\hline \hline & \multicolumn{4}{c}{ Elastic constant $k\left(\mathrm{eV} / \AA^{2}\right)$} \\
\cline { 2 - 3 } \cline { 5 - 6 } Element & \multicolumn{2}{c}{$[100]$} & & \multicolumn{2}{c}{$[111]$} \\
\cline { 2 - 3 } \cline { 5 - 7 } $\mathrm{Cu}$ & 3.56 & 4.84 & & $3.17(3.77)^{\mathrm{a}}$ & $6.01(6.1)^{\mathrm{b}}$ \\
$\mathrm{Au}$ & 3.32 & 3.86 & & $2.95(3.22)^{\mathrm{a}}$ & 6.13()$^{\mathrm{c}}$ \\
\hline \hline
\end{tabular}

${ }^{\mathrm{a}}$ From Ref. [42].

${ }^{\mathrm{b}}$ From Ref. [41].

${ }^{\mathrm{c}}$ Not available.

Note that for the case of $\mathrm{Au}$, the elastic constant of the adatom in the [100] surface is only slightly larger than for an atom of the surface, but the enhancement is much more significant in the [111] surface. Although there are no DFT results for a $\mathrm{Au}$ adatom, the comparison in the case of $\mathrm{Cu}$ is quite reasonable. In order to test if the high elastic constant of the adatom in the [111] surface changes the distance traces, we have performed simulations of $\mathrm{Cu}$ and $\mathrm{Au}$ with the same tips employed to obtain the results of Fig. 3 ([100] oriented), but this time on [111] surfaces, and with the same three configurations (tip to adatom, tip to surface atom and tip to hollow position on surface), as shown in Fig. 7. Interestingly, we still observe a sharp transition to contact in the case of $\mathrm{Au}$ and an adatom [Fig. 7(a)] and a smooth transition for $\mathrm{Cu}$ on an adatom [Fig. 7(b)]. The main difference is, as explained above, the relaxation of the $\mathrm{Au}$ tip, compared to the $\mathrm{Cu}$ one. In $\mathrm{Au}$, due to the high elastic constant of the adatom, this one moves less when the tip approaches the [111] surface than the [100] surface. According to our simulations, as well as previous models (Calvo [15], Untiedt et al. [3], and Trouwborst et al. [6]), the elastic properties of the material play a significant role in the presence or absence of this sharp transition.

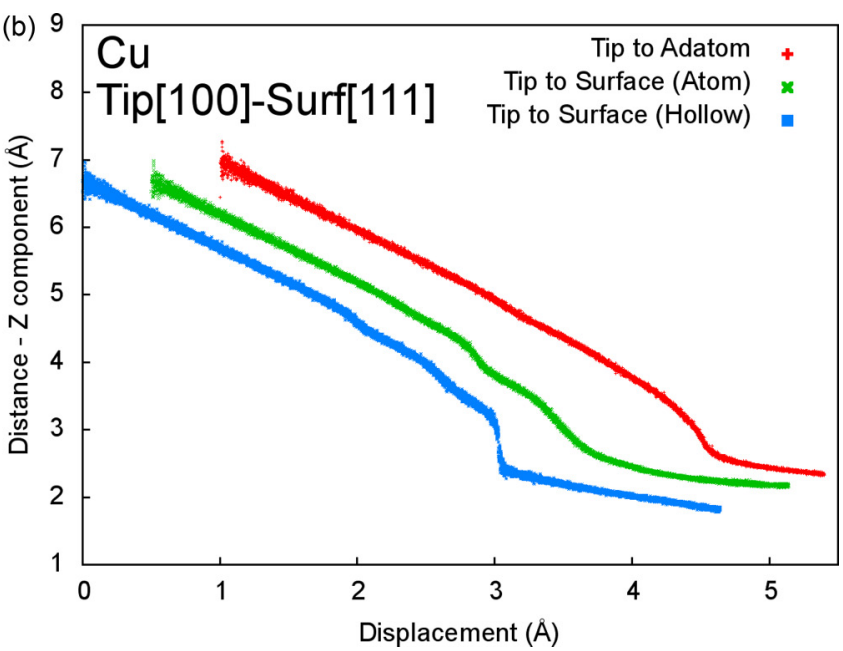

FIG. 7. CMD simulations for (a) $\mathrm{Au}$ and (b) $\mathrm{Cu}$ of [100] tips on [111] surfaces. Distance between electrodes ( $Z$ component) versus displacement (both in $\AA$ ) for a sharp tip approaching a surface. Three different scenarios: tip aligned with surface atom (green curve), tip aligned with hollow site (blue), and tip aligned with adatom lying in hollow (red). The traces have been shifted horizontally by $0.5 \AA$. 
According to Kröger et al. [4], 70\% to $80 \%$ of the time that there is a jump to contact when a tip approaches a flat surface, an adatom is found on the surface after retracting the tip. This suggested that, in these experiments, the most common mechanism occurring is that observed in our simulations when a $\mathrm{Cu}$ tip approaches a hollow site on a surface (Video 4) [26], that is, a detachment of the atom at the tip.

It is important to emphasize that the tips used in these simulations were constructed as almost perfectly pyramidal at the apex, with the last atoms lying in the fourfold hollow site created by four equally distributed atoms in its upper layer. We have also performed calculations of tips to surfaces using tips obtained from the mechanical annealing process explained above. In the case of $\mathrm{Au}$, and as a result of its elasticity, a sharp transition to contact is observed in all situations studied with these new tips. However, in $\mathrm{Cu}$, the geometry of the tip has a strong impact on how contact occurs: for very perfect tips, a smooth transition is observed when approaching a surface atom while a less perfect tip results in an abrupt transition to contact. Details of these results can be found in the supplemental material [26]. In the case of the mechanical annealing simulations presented here, the tips obtained, despite being very sharp tips, are in general more irregular than those artificially created by construction. This could explain why they always exhibit jump to contact, even in $\mathrm{Cu}$, in perfect agreement with MCBJ-like experiments. In $\mathrm{Cu}$, even a small irregularity in one of the tips gives rise to a sharp transition in the distance traces.

\section{CONCLUSIONS}

Through the analysis of thousands of conductance traces obtained experimentally for several metals during the process of contact between two tips, we obtained a correlation between the cohesive energy of the metal, its Young's modulus and the frequency of occurrence of a jump to contact. We observe that, for metals with low cohesive energies, a jump to contact almost always occurs. However, we also see that there is an influence of the Young's modulus: if the Young's modulus of the metal is high, the occurrence of jump to contact is less likely. These results imply that, in the process of bonding, not only local interactions between the last few atoms forming a nanojunction are important, but also longer-range interactions which could explain the correlation between bulk properties and the behavior at the nanoscale [27].

With the use of molecular dynamics simulations and electronic transport calculations we have shown that Au always undergoes a jump to contact, for any configuration of the tip and surface studied here, and also under mechanical annealing; that is, during cyclic loading of a tip on a surface. $\mathrm{Cu}$, on the other hand, shows jump to contact under mechanical annealing, as observed in MCBJ-like experiments [3,14,15], but it shows a smooth transition when a sharp tip approaches an adatom on the surface or a sharp tip directly on top of an atom in the surface. These results are in good agreement with the experimental observations of Kröger et al. [4,5] for $\mathrm{Cu}$ and $\mathrm{Au}$. Further experiments to confirm the correlations shown in Fig. 1 for other materials would be desirable. We should note that our simulations unveil two different mechanisms that result in a sharp change in the conductance (or the distance) between electrodes: in $\mathrm{Au}$, due to its soft nature, several atoms can relax during the approach. In $\mathrm{Cu}$, a more rigid material, the sharp transition (when employing very perfect tips) occurs due to the jumping of the atom at the tip apex to the surface.

The results described above can be understood in terms of the short-range cohesive forces and the longer-range elastic forces. When the material has a low Young's modulus, like in the case of $\mathrm{Au}$, atoms can relax easily and they will do so to achieve a high electronic density by increasing the number of neighbors, that is, decreasing the distance to their closest neighbors. As a consequence, when two tips of a material with low Young's modulus approach each other, atoms can relax easily to increase the embedding energy and an abrupt change at the point of contact is observed. This sharp change in distance corresponds to a sharp change in conductance, observed experimentally as a jump to contact. On the other hand, if the material is stiffer, such as for $\mathrm{Cu}$, and the apex of the tip is almost perfectly pyramidal, relaxation of atoms at the tip is limited, and such sharp jumps are not always possible. The contact then occurs through a smooth transition. However, even in the case of $\mathrm{Cu}$, for certain configurations, such as two sharp tips or less perfect tips approaching a flat surface, a sharp transition can be observed. This might also explain the difference between the observations of conductance obtained with two sharp tips in $\mathrm{Cu}$ and those of a sharp tip and an adatom.

We have shown that, for soft materials $(\mathrm{Au})$, the shape of the tip is not too relevant for the existence or absence of a JC (which is always present), while for stiffer materials $(\mathrm{Cu})$ the configuration of tip and surface plays a very important role in the transition from tunneling to contact regime. If nanocontacts are to be used as electrodes to probe electrical properties of molecules, for example, precise information about their stability, geometry, and behavior will be needed. These simulations provide a deeper understanding of how metals behave in the process of bonding, from atomic-scale processes to macroscopic properties.

\section{ACKNOWLEDGMENTS}

We thank Dr. J. Kröger and Professor van Ruitenbeek for their contributions. W. Dednam thanks Dr. A. E. Botha for helpful discussions and acknowledges support from the National Research Foundation of South Africa through the Scarce Skills Masters scholarship funding programme (Grant Unique Number 92138). This work is supported by the Generalitat Valenciana through Grant Reference PROMETEO2012/011 and MINECO under Grant No. FIS2013-47328, by European Union structural funds and the Comunidad de Madrid Programs S2013/MIT-3007 and P2013/MIT-2850. This work is also part of the research programme of the Foundation for Fundamental Research on Matter (FOM), which is financially supported by the Netherlands Organisation for Scientific Research (NWO). Some of the calculations in this paper were performed on the high-performance computing (HPC) facility at the University of South Africa's Muckleneuk campus in Pretoria, South Africa. The authors thankfully acknowledge the computer resources, technical expertise, and assistance provided by the Centro de Computacion Científica of the Universidad Autónoma de Madrid. 
[1] G. Binnig, H. Rohrer, C. Gerber, and E. Weibel, Phys. Rev. Lett. 49, 57 (1982).

[2] M. Crommie, C. Lutz, D. Eigler, and E. Heller, Surf. Rev. Lett. 02, 127 (1995).

[3] C. Untiedt, M. J. Caturla, M. R. Calvo, J. J. Palacios, R. C. Segers, and J. M. van Ruitenbeek, Phys. Rev. Lett. 98, 206801 (2007).

[4] J. Kröger, N. Néel, and L. Limot, J. Phys.: Condens. Matter 20, 223001 (2008).

[5] J. Kröger, N. Néel, A. Sperl, Y. F. Wang, and R. Berndt, New J. Phys. 11, 125006 (2009).

[6] M. L. Trouwborst, E. H. Huisman, F. L. Bakker, S. J. van der Molen, and B. J. van Wees, Phys. Rev. Lett. 100, 175502 (2008).

[7] M. P. Allen and D. J. Tildesley, Computer Simulation of Liquids, reprint ed., Oxford Science Publications (Oxford University Press, USA, 1989).

[8] N. Agraït, A. Levy Yeyati, and J. M. van Ruitenbeek, Phys. Rep. 377, 81 (2003).

[9] P. Hohenberg and W. Kohn, Phys. Rev. 136, B864 (1964).

[10] M. R. Sørensen, M. Brandbyge, and K. W. Jacobsen, Phys. Rev. B 57, 3283 (1998).

[11] C. Sabater, C. Untiedt, J. J. Palacios, and M. J. Caturla, Phys. Rev. Lett. 108, 205502 (2012).

[12] C. Sabater, M. Caturla, J. Palacios, and C. Untiedt, Nanoscale Res. Lett. 8, 257 (2013).

[13] C. Untiedt, G. Rubio, S. Vieira, and N. Agraï, Phys. Rev. B 56, 2154 (1997).

[14] C. Sabater, Ph.D. thesis, school Universidad de Alicante, 2013.

[15] M. R. Calvo, Ph.D. thesis, Universidad de Alicante, 2009.

[16] Wolfram Research, Young's Modulus of the Elements, available at http://www.periodictable.com/Properties/A/YoungModulus. html.

[17] G. Kaptay, G. Csicsovszki, and Y. M. S., Mater. Sci. Forum 414-415, 235 (2003).

[18] M. S. Daw and M. I. Baskes, Phys. Rev. Lett. 50, 1285 (1983).

[19] X. Zhou, H. Wadley, R. Johnson, D. Larson, N. Tabat, A. Cerezo, A. Petford-Long, G. Smith, P. Clifton, R. Martens, and T. Kelly, Acta Mater. 49, 4005 (2001).

[20] S. Plimpton, J. Comput. Phys. 117, 1 (1995).

[21] S. Plimpton et al., LAMMPS Molecular Dynamics Simulator, available at http://lammps.sandia.gov/.

[22] S. Nosé, Mol. Phys. 52, 255 (1984).

[23] W. G. Hoover, Phys. Rev. A 31, 1695 (1985).
[24] T. N. Todorov and A. P. Sutton, Phys. Rev. Lett. 70, 2138 (1993).

[25] J. Kröger, H. Jensen, and R. Berndt, New J. Phys. 9, 153 (2007).

[26] See Supplemental Material at http://link.aps.org/supplemental/ 10.1103/PhysRevB.93.085437 for details on mechanical annealing simulation procedure, calculation of minimum breaking force of a gold nanocontact, and simulation of tips from mechanical annealing on surfaces. Additionally, there can be seen a few short videos, showing the moment of contact in some tip-to-surface approximations.

[27] W. Dednam, C. Sabater, M. A. Fernandez, C. Untiedt, J. J. Palacios, and M. J. Caturla, J. Phys.: Conf. Ser. 574, 012045 (2015).

[28] G. Rubio, N. Agraït, and S. Vieira, Phys. Rev. Lett. 76, 2302 (1996).

[29] J. J. Palacios, A. J. Pérez-Jiménez, E. Louis, and J. A. Vergés, Phys. Rev. B 64, 115411 (2001).

[30] J. J. Palacios, A. J. Pérez-Jiménez, E. Louis, E. SanFabián, and J. A. Vergés, Phys. Rev. B 66, 035322 (2002).

[31] E. Louis, J. A. Vergés, J. J. Palacios, A. J. Pérez-Jiménez, and E. SanFabián, Phys. Rev. B 67, 155321 (2003).

[32] J. J. Palacios et al., Computer Code ANT.G, available at http://www.dfa.ua.es/en/invest/condens/Alacant/.

[33] M. J. Frisch et al., Gaussian09 Revision A.01 (Gaussian Inc., Wallingford, 2009).

[34] G. M. Finbow, R. M. Lynden-Bell, and I. R. McDonald, Mol. Phys. 92, 705 (1997).

[35] A. Stukowski, Modell. Simul. Mater. Sci. Eng. 18, 015012 (2010).

[36] A. Stukowski, OVITO. The Open Visualisation Tool, available at http://ovito.org/.

[37] E. Scheer, N. Agrait, J. C. Cuevas, A. L. Yeyati, B. Ludoph, A. Martin-Rodero, G. R. Bollinger, J. M. van Ruitenbeek, and C. Urbina, Nature (London) 394, 154 (1998).

[38] J. M. Krans, C. J. Muller, I. K. Yanson, T. C. M. Govaert, R. Hesper, and J. M. van Ruitenbeek, Phys. Rev. B 48, 14721 (1993).

[39] C. Sirvent, J. G. Rodrigo, S. Vieira, L. Jurczyszyn, N. Mingo, and F. Flores, Phys. Rev. B 53, 16086 (1996).

[40] U. Landman, W. D. Luedtke, N. A. Burnham, and R. J. Colton, Science 248, 454 (1990).

[41] L. Limot, J. Kröger, R. Berndt, A. Garcia-Lekue, and W. A. Hofer, Phys. Rev. Lett. 94, 126102 (2005).

[42] W. Hofer, A. Garcia-Lekue, and H. Brune, Chem. Phys. Lett. 397, 354 (2004). 